\title{
ARTICLE
}

Molecular Diagnostics

\section{A minority-group of renal cell cancer patients with high infiltration of CD20+B-cells is associated with poor prognosis}

\author{
Elin Sjöberg ${ }^{1}$, Magnus Frödin ${ }^{1}$, John Lövrot ${ }^{1}$, Artur Mezheyeuski $^{2}$, Martin Johansson ${ }^{3}$, Ulrika Harmenberg ${ }^{1}$, Lars Egevad $^{1}$, \\ Per Sandström ${ }^{1}$ and Arne Östman ${ }^{1}$
}

\begin{abstract}
BACKGROUND: The role of B-lymphocytes in solid tumours is unclear. Tumour biology studies have implied both anti- and protumoural effects and prognostic studies have mainly linked B-cells to increased survival. This study aimed to analyse the clinical relevance of B-lymphocytes in renal cell cancer (RCC), where information on the prognostic impact is lacking.

METHODS: Following immunohistochemistry (IHC) stainings with a CD20 antibody, density of CD20+ B-cells was quantified in an RCC discovery- and validation cohort. Associations of B-cell infiltration, determined by CD20 expression or a B-cell gene-signature, and survival was also analysed in 14 publicly available gene expression datasets of cancer, including the kidney clear cell carcinoma (KIRC) dataset.

RESULTS: IHC analyses of the discovery cohort identified a previously unrecognised subgroup of RCC patients with high infiltration of CD20+ B-cells. The B-cell-high subgroup displayed significantly shorter survival according to uni- and multi-variable analyses. The association between poor prognosis and high density of CD20+ B-cells was confirmed in the validation cohort. Analyses of the KIRC gene expression dataset using the B-cell signature confirmed findings from IHC analyses. Analyses of other gene expression datasets, representing 13 different tumour types, indicated that the poor survival-association of B-cells occurred selectively in RCC. CONCLUSION: This exploratory study identifies a previously unrecognised poor-prognosis subset of RCC with high density of CD20defined B-cells.
\end{abstract}

British Journal of Cancer (2018) 119:840-846; https://doi.org/10.1038/s41416-018-0266-8

\section{INTRODUCTION}

Renal cell cancer (RCC) originates from the renal epithelial cells and constitutes $\sim 3$ percent of all human malignancies. ${ }^{1}$ Despite advancements in targeted therapies in the last decade, the disease is notoriously difficult to treat once metastasis is manifest, with an 5-year survival rate of only 8-12 percent. $^{2}$ The most common form of RCC is the clear cell type, which make up $~ 70 \%$ of all RCC, and is characterised by an inactivation of the vonHippel-Lindau gene. ${ }^{3}$ Anti-angiogenic drugs currently used for the treatment of metastatic RCC are based on clinical trials with sunitinib, pazopanib, axitinib and cabozantinib. ${ }^{4,5}$ More recently, immune checkpoint regulators have been added to the armamentarium, ${ }^{6}$ although the mechanism by which they exert their antitumoural function in RCC is not fully understood.

The immune system has during recent years been an attractive target for treatment of several cancers. T-cells are the best characterised immune cells that have an antitumour function and correlate with a favourable prognosis in many tumours. ${ }^{7,8}$ On the contrary, B-lymphocytes are less studied and the role during tumour progression is not fully understood. Also, clinical relevance of these findings remains largely unknown. Some recent studies have reported tumour promoting B-cells in tumours. These tumour promoting functions involve modulation of
T-cell-mediated tumour cell killing and responses to chemotherapy $^{9,10}$ or secretion of tumour stimulatory cytokines. ${ }^{11,12}$

Tumour suppressive functions of B-lymphocytes are implied by a number of studies which have identified an association of B-cells and prolonged survival in different cancers, including liver cancer, ovarian cancer, pancreatic adenocarcinoma, melanoma, colorectal cancer, oesophageal cancer, gastric cancer and breast cancer. ${ }^{13-21}$

In some cancers, the association between B-lymphocytes and prognosis is less clear and studies have also reported that B-cells is associated with worse outcome. In patients with primary cutaneous melanoma and ovarian cancer CD20+ and CD138+ Bcells, respectively, correlated to decreased overall survival (OS). ${ }^{15,22}$ In a study by Mahmoud et al., the number of CD20expressing B-cells in breast tumours was associated with higher tumour grade, hormone receptor negativity and increased breast cancer specific survival. ${ }^{20}$ In a recent study of ductal carcinoma in situ (DCIS), presence of B-lymphocytes also correlated with hormone receptor negativity and large tumour size. However, patients with high B-lymphocyte infiltration had a shorter recurrence free survival. ${ }^{23}$

An extensive analysis of the immune landscape in clear cell RCC was recently published, where a specific immune signature derived from single cell analysis of human tumours correlated to

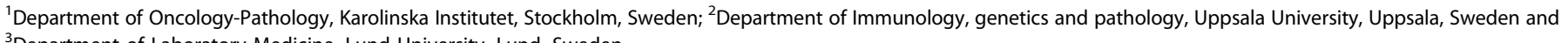
${ }^{3}$ Department of Laboratory Medicine, Lund University, Lund, Sweden

Correspondence: Arne Östman (arne.ostman@ki.se)

These authors contributed equally: Per Sandström, Arne Östman

Received: 5 March 2018 Revised: 15 August 2018 Accepted: 31 August 2018

Published online: 8 October 2018 
shorter progression free survival. ${ }^{24}$ Associations between B-cell infiltration and survival in RCC have not earlier been reported. In this study we identify a correlation between CD20-defined B-cells, determined by IHC, and worse survival in two independent RCC cohorts. This association was also supported by analyses of publicly available transcriptome data. Furthermore, we show that the association of CD20 expression and poor prognosis appear selective to RCC.

\section{MATERIAL AND METHODS}

Patient material and tissue microarrays

Two RCC tissue microarrays (TMAs) were used in the study.

The discovery cohort is a population-based cohort consisting of 314 RCC patients diagnosed between 1978 and 1996 at Skåne University Hospital Malmö, Sweden. For validation, a second TMA was made from 64 RCC patients diagnosed between 1997 and 2005 at Karolinska University Hospital, Stockholm, Sweden, who all developed metastatic disease which was treated with at least one course of sunitinib as first line treatment. M-stage in this cohort refers to presence of metastasis at diagnosis. Data of patient characteristics, treatment and OS was collected in clinical registries. Details about the cohorts are presented in Table 1. The discovery cohort has been used in previous biomarker studies. ${ }^{25,26}$ For both TMAs tumours were revised by a pathologist and representative tumour parts were chosen. Each tumour was represented by two $1 \mathrm{~mm}$ diameter core punch biopsies.

\section{Immunohistochemistry staining and evaluation}

Tumour sections were deparaffinised and rehydrated in xylene, 99\% ethanol, $95 \%$ ethanol and $70 \%$ ethanol, and then washed in distilled water. Antigen retrieval was obtained in a decloaking chamber (Biocare Medical) at $95^{\circ} \mathrm{C}$ for $15 \mathrm{~min}$ in $\mathrm{pH} 9.0$ retrieval buffer (Dako, Cat. nr. S2367) followed by cooling in room temperature for $30 \mathrm{~min}$. Endogenous peroxidase was quenched by incubation with $3 \% \mathrm{H}_{2} \mathrm{O}_{2}$ (Invitrogen, Stockholm, Sweden) for 10 min. After washing in PBS with $0.1 \%$ Tween 20 , tumour sections were blocked in Protein Block (Dako, Serum-Free Ready to Use, Cat. $\mathrm{nr}$ X0909) for $1 \mathrm{~h}$ at room temperature. As primary antibody, CD20 mouse anti-human antibody (Dako, Cat. nr M0755) was used at a concentration of 1:300 and tissue slides were incubated over night at $4{ }^{\circ} \mathrm{C}$. Thereafter, the secondary antibody (HRP-conjugated goat anti-mouse, Dako, Cat. nr. P0447) was added for $1 \mathrm{~h}$ at room temperature. For antibody detection, slides were incubated with DAB (Vector Laboratories, Burlingame, CA, USA) for 2 min and counterstaining with haematoxylin (Histolab Products $A B$, Gothenburg, Sweden) was performed for $30 \mathrm{~s}$, followed by dehydration and mounting with Vectamount permanent mounting media (Vector Laboratories, Inc., Burlingame, CA, USA).

The CD20-stainings were evaluated by two investigators (ES and MF), mentored by board-certified pathologist (LE), and performed blinded with regard to outcome and clinic-pathological characteristics. Each case was assigned a score based on the mean number of $\mathrm{CD} 20+$ cells detected in each of the two tissue cores from each case.

Analyses of gene expression data sets

The association of B-cell infiltration and survival was analysed in a clinical cohort of clear cell RCC with publicly available transcriptome data (KIRC), generated by The Cancer Genome Atlas (TCGA) Research Network (http://cancergenome.nih.gov/). The dataset consists of gene expression data from 534 patients with clear cell RCC. B-cell infiltration was assessed by a B-cell gene expression signature score created by log2-transformation of the Z-score values for CD19, MS4A1 and PAX5 in KIRC, obtained from cbioportal. The same cut-off (86-percentile) as for the discovery cohort was used for dichotomisation of patients with low or high B-cell infiltration.
Publicly available gene expression datasets from 14 cancer types from the TCGA database was used to analyse the association between the gene expression of MS4A1 (CD20) and survival.

\section{Statistical analyses}

For determination of the cut-off value for dichotomisation, The $\mathrm{R}$ package flexmix was used to fit a zero-inflated Poisson mixture model of CD20 data in the discovery cohort. The model is a mixture of two Poission distributions (low and high abundance of CD20-positive cells) and a point distribution at zero. A cut-point for dichotomisation into low and high abundance was determined based on the posterior probabilities. ${ }^{27}$

Association of $\mathrm{CD} 20+$ staining or the B-cell signature with clinic-pathological parameters was analysed with Fisher exact test or Pearson Chi-square test.

The duration of survival time was calculated from the date of diagnosis to the date of death or last known follow-up. Probabilities of survival were estimated using the Kaplan-Meier method and log-rank test. The correlation of CD20 status with outcome was evaluated using Cox proportional hazards regression model in uni- and multi-variable analyses.

Statistical analyses were done using the SPSS software package 21.0 (IBM Corporation, Armonk, NY). P-values <0.05 were considered statistically significant.

\section{RESULTS}

A minority-group of RCC patients display high infiltration of CD20+ B-cells

Exploratory analyses were performed to characterise inter-case variations with regard to infiltration of B-cells, as determined by IHC staining of CD20, in a population-based cohort of RCC (referred to as the discovery cohort).

Of the 314 RCC patients included in the TMA, 297 yielded informative staining. Of these 297 patients, the large majority displayed no infiltration or low infiltration of CD20+ B-lymphocytes (Fig. 1, left and middle panel). However, a minority group of patients was detected with prominent infiltration of CD20+ cells (Fig. 1, right panel). Statistical analysis of the distribution of CD20+ B-cells among patients established a cut-off of 16 cells/core for division of patients into two groups (Supp. Figure 1 and Material and Methods for details). This cut-off generated a subgroup of patients with high Bcell infiltration composed of $14 \%$ of the population.

Initial analyses to explore the clinical relevance demonstrated that the B-cell-high subgroup was significantly associated with high Fuhrman grade $(p$-value $=0.007)$ and metastasis $(p$-value $=$ 0.034 ), but no associations were detected between B-cell-status and sex, age, tumour stage or histology (Table 1).

Together these analyses identify a previously unrecognised subset of RCC patients with high infiltration of CD20 + B-cells.

High infiltration of B-cells is an independent poor prognosis marker in renal cancer

The data on tumour infiltrating CD20+B-cells were combined with patient survival data to explore the potential correlation between CD20-status and outcome.

As shown in Fig. 2, Kaplan-Meier analysis demonstrated that the B-cell-high group displayed significantly shorter OS ( $p$-value < 0.001; Log-rank-test) (Fig. 2a). Uni-variable Cox-regression analysis confirmed these findings and revealed and increased risk of cancer related death for patients with high infiltration of B-cells $(\mathrm{HR}=1.98 ; 95 \% \mathrm{Cl}=1.32-2.96 ; p$-value $<0.001)$.

Furthermore, multi-variable analyses, also including sex, age, histology, T-stage, M-stage and Fuhrman-grade, demonstrated independent prognostic significance of B-cell status in RCC patients $(\mathrm{HR}=2.7 ; 95 \% \mathrm{Cl}=1.3-5.6 ; p$-value $=0.008)$ (Table 2$)$.

To validate these findings, CD20-status was determined in an independent cohort of RCC (referred to as the validation cohort) 
A minority-group of renal cell cancer patients with high infiltration of...

E. Sjöberg et al.

Table 1. Associations between CD20-status and clinicopathological parameters in the discovery cohort, validation cohort and KIRC

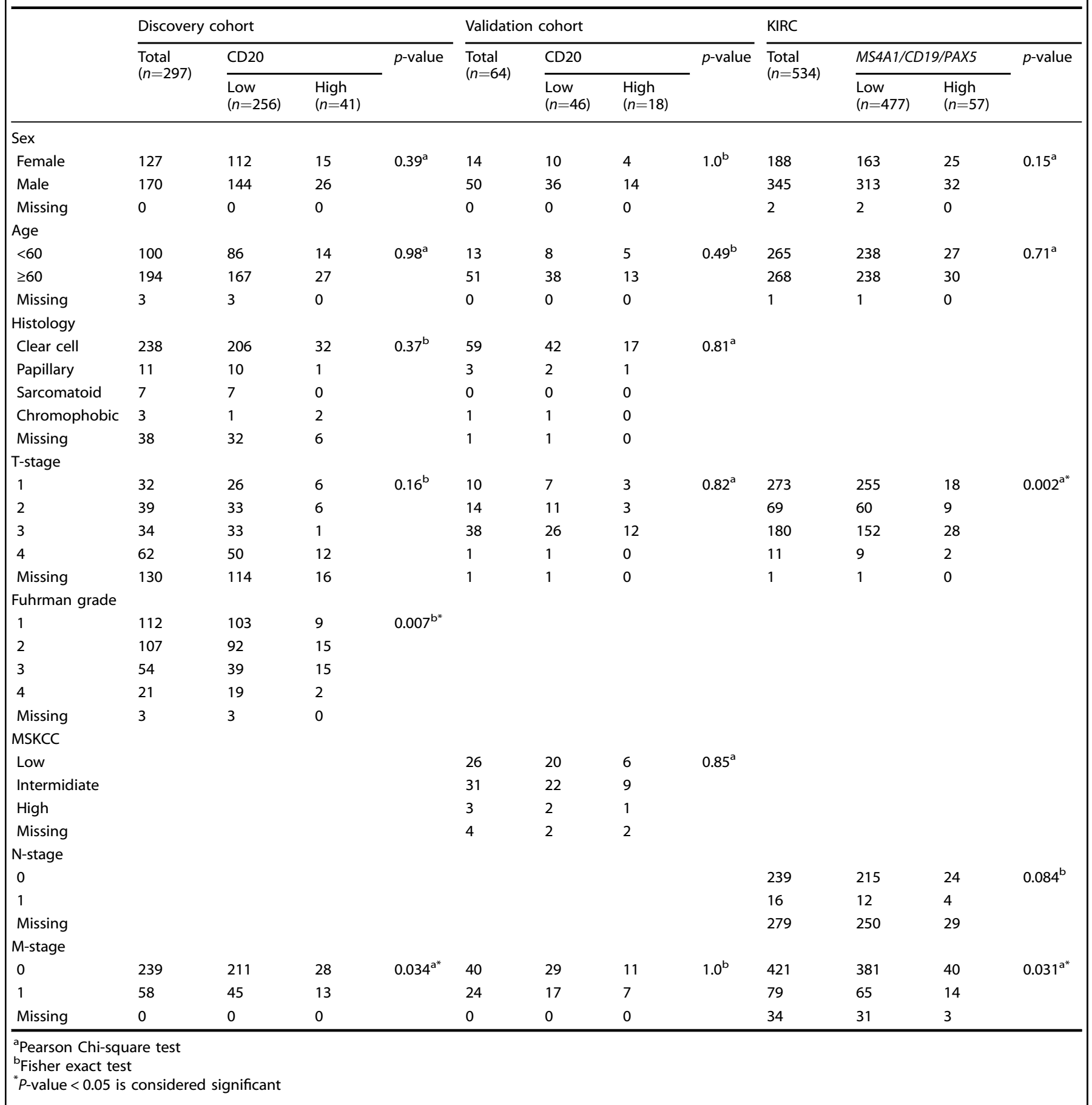

composed of patients, which at metastatic stage had been treated with sunitinib (see Material and Methods for details). As in the population-based discovery cohort a minority B-cell-high group (28\%) was identified also in this cohort, using the same cut-off (16 cells/core). In this cohort, B-cell-status was not significantly associated with any clinic-pathological parameters including sex, age, histology, MSKCC-grade, T- or M-stage (Table 1).

Survival analyses in the validation cohort confirmed a significant association between shorter OS and B-cell-high status in RCC patients as shown in Fig. $2 \mathrm{~b}$ ( $p$-value $=0.034$; Log-Rank-test), and an increased risk of death in the CD20-high group as determined by uni-variable Cox Regression analysis $(\mathrm{HR}=1.9 ; 95 \% \mathrm{Cl}=$
1.0-3.6; $p$-value $=0.039$ ). Furthermore, $C D 20$-status demonstrated independent prognostic significance in multi-variable analyses including clinico-pathological characteristics (HR 2.9; $95 \% \mathrm{Cl}=1.4$ - 6.4; $p$-value $=0.005$ ) (Table 3).

Together these analyses, using two different patient cohorts, identify infiltration of CD20 + B-cells as a novel independent marker for RCC survival.

A B-cell signature is correlated with poor prognosis in the TCGA clear cell RCC cohort

To extend the IHC-based analyses, the clinical impact of a B-cell infiltration was analysed in the TCGA gene expression dataset of 

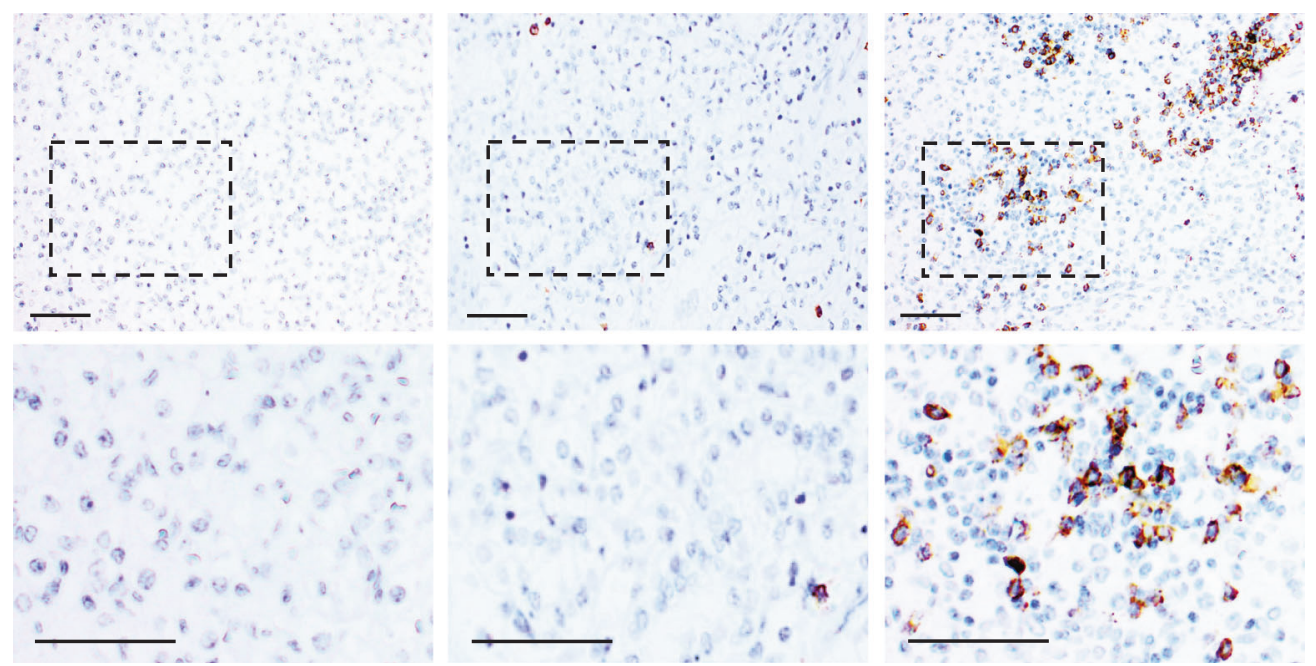

Fig. 1 Variable infiltration of CD20+ cells in RCC. Immunohistochemistry staining with CD20 antibodies was used to identify tumour infiltrating B-lymphocytes in RCC patients. The majority of patients displayed no (left panel) or low infiltration (middle panel), whereas a minority of cases demonstrated high infiltration of CD20+ cells (right panel). Scale bar $100 \mu \mathrm{m}$

clear cell RCC (KIRC). B-lymphocytes express additional markers besides CD20. Other well-established markers for B-cells are CD19 and the B-cell transcription factor PAX5. ${ }^{28}$

We therefore composed a B-cell gene signature composed of MS4A1 (CD20), CD19 and PAX5, and a signature score was established for each patient. Based on the dichotomisation of the discovery cohort described above, a patient group of high Bcell signature score was defined composed of the $14 \%$ of cases with highest score. Initial analyses demonstrated that this subgroup of patients with high B-cell signature displayed significant positive association with tumour stage ( $p$-value $=$ $0.002)$ and metastasis ( $p$-value $=0.031)($ Table 1$)$.

Survival analyses demonstrated a significantly shorter OS in the B-cell-signature-high group as determined by Log-Rank-test (Fig. 3a; $p$-value $=0.042$ ) and uni-variable Cox-regression analyses $(\mathrm{HR}=1.54 ; \mathrm{Cl}=1.01-2.33 ; p$-value $=0.043)$. The B-cell-signature did not remain significant in multivariable analysis including sex, age, $\mathrm{T}-\mathrm{N}$ - and $\mathrm{M}$-stage (Table 3 ).

This signature-based analysis thus supports findings from the IHC analyses indicating the existence of a minority-group of RCC with high B-cell-infiltration and poor prognosis.

MS4A1-expression is not associated with poor prognosis in other common types of cancer

The general understanding that B-lymphocytes are part of an antitumour immune response has during recent years been challenged. However, prognostic studies supporting a clinical relevant tumour promoting effect of B-cells is limited.

We therefore performed screening-like exploratory analyses of potential survival associations of high B-cell infiltration, defined by high expression of the gene for CD20 (MS4A1), in other tumour types through analyses of a selection of 14 publicly available datasets (including KIRC). For these analyses the minority B-cellhigh groups were defined as the $10 \%$ of cases with highest MS4A1 expression.

In agreement with previous findings, the MS4A1-high group in RCC showed a significant association with poor survival $(H R=$ $1.63 ; \mathrm{Cl}=1.03-2.59 ; \mathrm{p}$-value $=0.039$ ) (Fig. 3b). In most cohorts, no significant associations were detected between MS4A1-status and survival (Fig. 3b). Notably, high MS4A1expression was associated with good prognosis in cervical cancer, head and neck squamous cell carcinoma (HNSCC) and lung adenocarcinoma (Fig. 3b).

Collectively, these studies thus indicate that B-cells are associated with poor prognosis selectively in RCC.

\section{DISCUSSION}

This exploratory study of two independent RCC collections identifies a previously unrecognised minority-subset of RCC defined by high infiltration of CD20+B-cells, which is associated with poor prognosis. The existence of this subset is further supported by analyses of the TCGA clear cell RCC gene expression dataset, which confirmed an association between poor prognosis and high expression of either the gene for CD20 or a three-gene B-cell signature. Moreover, the poor prognoses signal of CD20expressing B-cells was exclusively found in RCC. The cases of the large discovery cohort of the present study did not receive any anti-angiogenic drugs. The survival associations of this study are thus likely reflecting aspects of the natural course biology of RCC.

These correlative studies suggest the possibility of a subset of RCC where B-cells exert pro-tumoural functions. Model-based studies have suggested numerous mechanisms whereby B-cells can stimulate tumour growth and alter response to therapy. These include production of autoantibodies, complement conjugation and secretion of immune-regulatory cytokines that affect macrophage and T-cell responses. ${ }^{12}$

In a mouse model of squamous carcinoma, CD20+ B-lymphocytes affect tumour growth and decrease response to chemotherapy by altering a macrophage dependent T-cell response. ${ }^{9}$ In line with this, targeting of B-cells in a mouse model of pancreatic cancer modulated macrophage function, restored tumour killing by T-cells and improved the response to chemotherapy. ${ }^{10}$

Some of the tumour promoting effects has been assigned to specific B-cell subsets. A recent study on pancreatic ductal adenocarcinoma identified a B-cell subpopulation that supported early tumour growth by secretion of IL-35. ${ }^{11}$ Additional tumour stimulatory subsets of B-lymphocytes characterised by PD1 expression or CXCL13 secretion have also been identified in mice. $^{29,30}$

CD20 and CD19 are considered pan-markers for B-cells. ${ }^{12}$ Future analyses of the marker- and gene expression status of the B-cells in the poor-prognosis subset of RCC should therefore give some guidance about the possible relevance of the above described mechanisms for the observed survival-associations in this study. Continued characterisation of B-cell subpopulations across tumour types might also help understanding the mechanisms behind the observed selective poor-prognosis association of CD20+B-lymphocytes in RCC.

The present analyses have not addressed to what extent the Bcell-high subgroup is strongly correlated to specific genetic or 

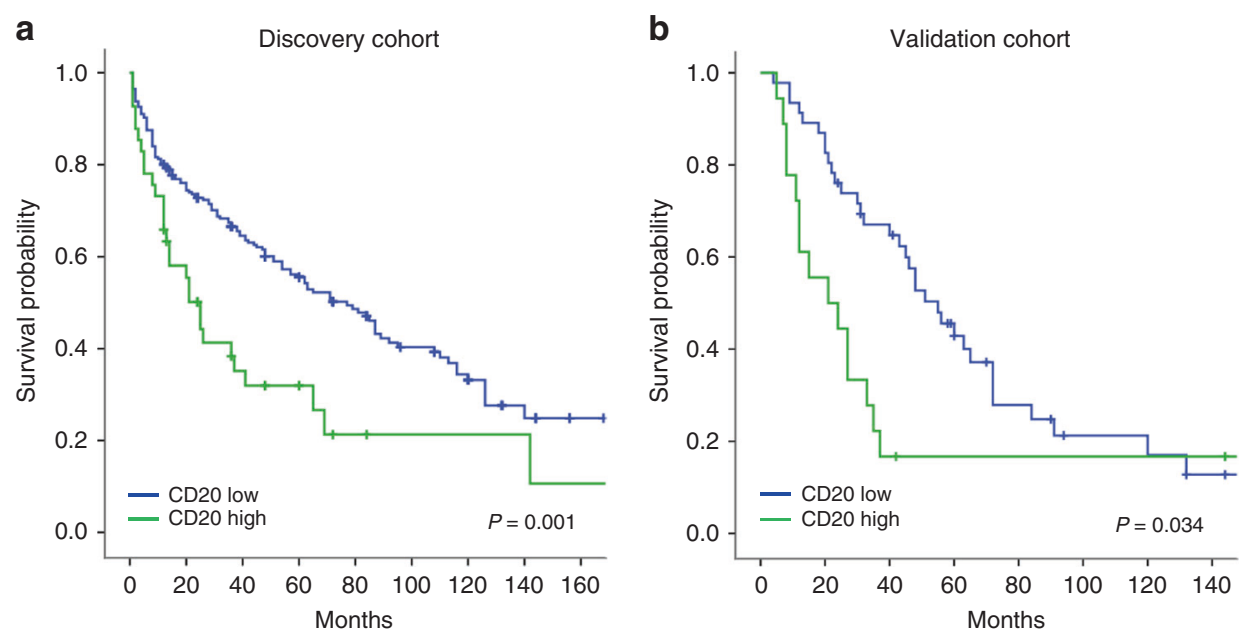

Fig. 2 Correlation of CD20 status and prognosis of RCC patients in two independent cohorts. Kaplan-Meier curves showing the overall survival of RCC patients with low or high infiltration of CD20+ cells in the discovery cohort (left panel) and the validation cohort (right panel)

\begin{tabular}{|c|c|c|c|}
\hline & HR & $95 \% \mathrm{Cl}$ & $p$-value \\
\hline \multicolumn{4}{|l|}{ Sex } \\
\hline Female & 1 & & \\
\hline Male & 0.8 & $0.4-1.3$ & 0.32 \\
\hline \multicolumn{4}{|l|}{ Age } \\
\hline$<60$ & 1 & & \\
\hline$\geq 60$ & 2.6 & $1.4-4.8$ & $0.002^{*}$ \\
\hline \multicolumn{4}{|l|}{ Histology } \\
\hline Non clear cell & 1 & & \\
\hline Clear cell & 0.2 & $0.1-0.5$ & $<0.001^{*}$ \\
\hline \multicolumn{4}{|l|}{ T-stage } \\
\hline 1 & 1 & & \\
\hline 2 & 1.4 & $0.4-5.1$ & 0.65 \\
\hline 3 & 1.8 & $0.5-7.1$ & 0.38 \\
\hline 4 & 3.9 & $1.1-13.9$ & $0.03^{*}$ \\
\hline \multicolumn{4}{|l|}{ Fuhrman grade } \\
\hline 1 & 1 & & \\
\hline 2 & 1.6 & $0.8-3-1$ & 0.2 \\
\hline 3 & 1.6 & $0.7-3.3$ & 0.2 \\
\hline 4 & 3.5 & $1.3-9.2$ & $0.01^{*}$ \\
\hline \multicolumn{4}{|l|}{ M-stage } \\
\hline 0 & 1 & & \\
\hline 1 & 5.7 & $3.1-10.7$ & $<0.001^{*}$ \\
\hline \multicolumn{4}{|l|}{ CD20+B-cells } \\
\hline Low & 1 & & \\
\hline High & 2.7 & $1.3-5.6$ & $0.008^{*}$ \\
\hline
\end{tabular}

epigenetic changes, to certain gene expression signatures or to overall mutation load. Further analyses of the TCGA RCC dataset should be productive in this regard. Possibly such studies can also shed light on the presently unexplained RCC-selectivity of the CD20-high survival association.

Immunotherapy with T-cell modulating antibodies is emerging as a new treatment option in RCC. ${ }^{31}$ Future work should consider the possibility that the B-cell-high RCC-subgroup displays particular response patterns to these treatments. Similarly, possible relationships to sensitivity to anti-
Table 3. Multivariable analysis for overall survival in the validation cohort and KIRC

\begin{tabular}{|c|c|c|c|c|c|c|c|}
\hline \multicolumn{4}{|c|}{ Validation cohort } & \multicolumn{4}{|l|}{$\mathrm{KIRC}$} \\
\hline & $\mathrm{HR}$ & $95 \% \mathrm{Cl}$ & $p$-value & & $\mathrm{HR}$ & $95 \% \mathrm{Cl}$ & $\mathrm{p}$-value \\
\hline Sex & & & & Sex & & & \\
\hline Female & 1 & & & Female & 1 & & \\
\hline Male & 0.9 & $0.4-2.2$ & 0.9 & Male & 1.2 & $0.8-1.8$ & 0.5 \\
\hline Age & & & & Age & & & \\
\hline$<60$ & 1 & & & $<60$ & 1 & & \\
\hline$\geq 60$ & 0.5 & $0.2-1.2$ & 0.1 & $\geq 60$ & 2.0 & $1.3-3.3$ & $0.002^{*}$ \\
\hline \multicolumn{8}{|l|}{ Histology } \\
\hline $\begin{array}{l}\text { Non clear } \\
\text { cell }\end{array}$ & 1 & & & & & & \\
\hline Clear cell & 2.1 & $0.6-7.9$ & 0.3 & & & & \\
\hline T-stage & & & & T-stage & & & \\
\hline 1 & 1 & & & 1 & 1 & & \\
\hline 2 & 1 & $0.3-3.0$ & 0.9 & 2 & 0.8 & $0.4-1.7$ & 0.5 \\
\hline 3 & 2.7 & $1.0-7.1$ & $0.04^{*}$ & 3 & 2.0 & $1.2-3.4$ & $0.007^{*}$ \\
\hline 4 & 0.9 & $0.1-8.8$ & 0.9 & 4 & 2.2 & $0.6-7.9$ & 0.2 \\
\hline MSKCC & & & & $\mathrm{N}$-stage & & & \\
\hline Low & 1 & & & 0 & 1 & & \\
\hline Intermediate & 2.9 & $0.8-3-1$ & $0.05^{*}$ & 1 & 2.5 & $0.9-6.3$ & 0.06 \\
\hline High & 5.3 & $0.7-3.3$ & $0.021^{*}$ & & & & \\
\hline M-stage & & & & M-stage & & & \\
\hline 0 & 1 & & & 0 & 1 & & \\
\hline 1 & 1.9 & $0.9-4.1$ & 0.1 & 1 & 3.3 & $1.9-5.5$ & $0.001^{*}$ \\
\hline CD20+B-cells & & & & MS4A1/CL & $19 / \mathrm{P}$ & $x 5$ & \\
\hline Low & 1 & & & Low & 1 & & \\
\hline High & 2.9 & $1.4-6.4$ & $0.005^{*}$ & High & 0.6 & $0.31-1.3$ & 0.2 \\
\hline
\end{tabular}

angiogenic treatments should also be explored. Future studies on well-matched groups, ideally derived from clinical trials, with or without anti-angiogenic or immunotherapy treatment are therefore warranted to explore relationships between the B-cell marker and response to treatment. Other important tasks for future validation studies include refinement of scoring criteria and identification of optimal cut-offs for dichotomisation. Towards potential clinical utility of the marker, these efforts should aim for a combination of prognostic effect and high feasibility. 

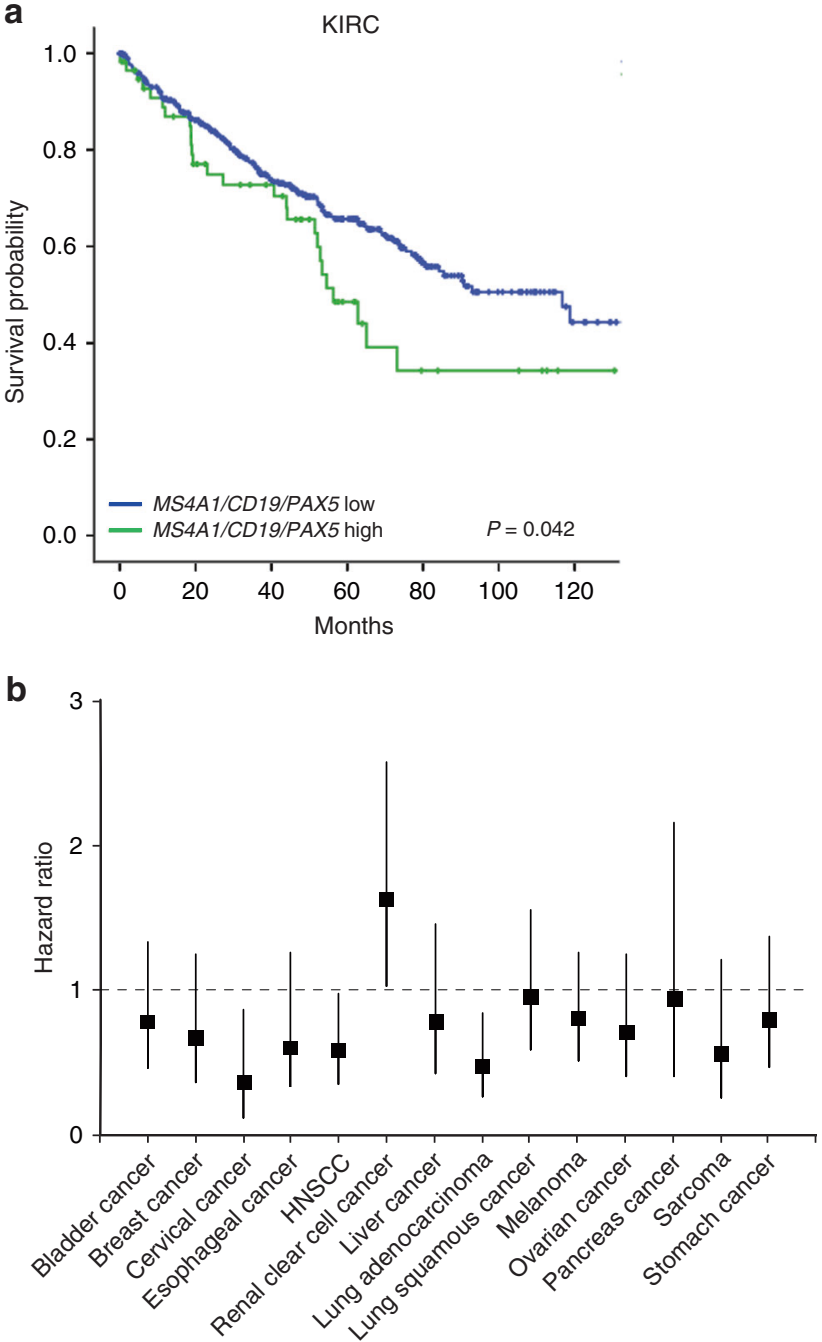

Fig. 3 Associations between survival and a B-lymphocyte signature or MS4A1 expression in gene expression datasets of different tumour types. a Kaplan-Meier plot showing overall survival of clear cell RCC patients in the KIRC gene expression dataset (TCGA) with low or high B-lymphocyte gene signature score (MS4A1/CD19/PAX5). b Association of MS4A1 expression and overall survival in 14 cancer gene expression datasets from the TCGA database

One notable implication of the observation that CD20+Blymphocytes is associated with worse survival of RCC patients, is that different CD20-directed therapies, presently approved for Bcell lymphomas ${ }^{32}$ can be considered as a treatment regimen for this novel subset of RCC. In this context novel mouse models of RCC should be useful tools for pre-clinical studies. ${ }^{3}$

In summary, findings from this exploratory study suggest future studies to be performed on larger clinically, genetically and molecularly well-annotated RCC cohorts. Such studies should provide additional information about the possibility to develop CD20-status to a biomarker of clinical utility for RCC. Equally important, such studies should also give further information on molecular and genetic features that are associated with CD20, and suggest mechanistic relationships that can have therapeutic implications.

\section{ACKNOWLEDGEMENTS}

Members of the AÖ-group are acknowledged for their support and constructive criticism throughout the study.

\section{AUTHOR CONTRIBUTIONS}

E.S. and M.F. conceived and carried out experiments. M.F., M.J., U.H., L.E. and P.S. were responsible for tissue collection and clinical databases. A.M. contributed to primary data analysis. A.Ö. and P.S. conceived experiments. All authors were involved in writing the paper and final approval of the submitted and published versions.

\section{ADDITIONAL INFORMATION}

Supplementary information is available for this paper at https://doi.org/10.1038/ s41416-018-0266-8.

Competing interests: The authors declare no competing interests.

Availability of data and material: The data and materials from the study are available upon request.

Ethics approval and consent to participate: All analysis of tumour tissue has been done in concordance with local and national ethical guidelines and legislations in Sweden. All patients have given their written informed consent and the studies was approved by the regional research ethics board in Stockholm (Dnr. 2010/1339 32) and the regional research ethics board in Lund (Dnr 282/2007). The study was performed in compliance with the 1975 Declaration of Helsinki.

Funding: During the study the AÖ-group was supported by grants from the Swedish Research Council (STARGET), the Breast Cancer Theme Center (BRECT) at Karolinska Institutet, Swedish Cancer Society, Radiumhemmets Forskningsfonder and Stockholm County Council (SLL).

Note: This work is published under the standard license to publish agreement. After 12 months the work will become freely available and the license terms will switch to a Creative Commons Attribution 4.0 International licence (CC BY 4.0).

\section{REFERENCES}

1. Hsieh, J. J. et al. Renal cell carcinoma. Nat. Rev. Dis. Primers 3, 17009 (2017)

2. Ghatalia, P., Zibelman, M., Geynisman, D. M. \& Plimack, E. R. Checkpoint inhibitors for the treatment of renal cell carcinoma. Curr. Treat. Options Oncol. 18, 7 (2017).

3. Harlander, S. et al. Combined mutation in Vhl, Trp53 and Rb1 causes clear cell renal cell carcinoma in mice. Nat. Med. 23, 869-877 (2017).

4. Choueiri, T. K. \& Motzer, R. J. Systemic therapy for metastatic renal-cell carcinoma. N. Eng. J. Med. 376, 354-366 (2017).

5. Choueiri, T. K. et al. Cabozantinib versus everolimus in advanced renal-cell carcinoma. N. Eng. J. Med. 373, 1814-1823 (2015).

6. Motzer, R. J. et al. Nivolumab for metastatic renal cell carcinoma: results of a randomized phase II trial. J. Clinical Oncology. 33, 1430-1437 (2015).

7. Fridman, W. H., Pages, F., Sautes-Fridman, C. \& Galon, J. The immune contexture in human tumours: impact on clinical outcome. Nat. Rev. Cancer 12, 298-306 (2012).

8. Vesely, M. D., Kershaw, M. H., Schreiber, R. D. \& Smyth, M. J. Natural innate and adaptive immunity to cancer. Annu. Rev. Immunol. 29, 235-271 (2011).

9. Affara, N. I. et al. B cells regulate macrophage phenotype and response to chemotherapy in squamous carcinomas. Cancer Cell. 25, 809-821 (2014).

10. Gunderson, A. J. et al. Bruton tyrosine kinase-dependent immune cell cross-talk drives pancreas cancer. Cancer Discov. 6, 270-285 (2016).

11. Pylayeva-Gupta, Y. et al. IL35-producing B cells promote the development of pancreatic neoplasia. Cancer Discov. 6, 247-255 (2016).

12. Gunderson, A. J. \& Coussens, L. M. B cells and their mediators as targets for therapy in solid tumors. Exp. Cell Res. 319, 1644-1649 (2013).

13. Shi, J. Y. et al. Margin-infiltrating $C D 20(+)$ B cells display an atypical memory phenotype and correlate with favorable prognosis in hepatocellular carcinoma. Clin. Cancer Res. 19, 5994-6005 (2013).

14. Nielsen, J. S. et al. CD20+tumor-infiltrating lymphocytes have an atypical CD27memory phenotype and together with $C D 8+T$ cells promote favorable prognosis in ovarian cancer. Clin. Cancer Res. 18, 3281-3292 (2012).

15. Lundgren, S., Berntsson, J., Nodin, B., Micke, P. \& Jirstrom, K. Prognostic impact of tumour-associated B cells and plasma cells in epithelial ovarian cancer. J. Ovarian Res. 9, 21 (2016).

16. Berntsson, J., Nodin, B., Eberhard, J., Micke, P. \& Jirstrom, K. Prognostic impact of tumour-infiltrating B cells and plasma cells in colorectal cancer. Int. J. Cancer 139, 1129-1139 (2016).

17. Ladanyi, A. et al. Prognostic impact of B-cell density in cutaneous melanoma. Cancer Immunol., Immunother. 60, 1729-1738 (2011). 
A minority-group of renal cell cancer patients with high infiltration of... E. Sjöberg et al.

846

18. Castino, G. F. et al. Spatial distribution of B cells predicts prognosis in human pancreatic adenocarcinoma. Oncoimmunology 5, e1085147 (2016).

19. Fristedt, R. et al. Prognostic impact of tumour-associated B cells and plasma cells in oesophageal and gastric adenocarcinoma. J. Gastrointest. Oncol. 7, 848-859 (2016).

20. Mahmoud, S. M. et al. The prognostic significance of $B$ lymphocytes in invasive carcinoma of the breast. Breast Cancer Res. Treat. 132, 545-553 (2012).

21. Sakimura, C. et al. B cells in tertiary lymphoid structures are associated with favorable prognosis in gastric cancer. J. Surg. Res. 215, 74-82 (2017).

22. Martinez-Rodriguez, M., Thompson, A. K. \& Monteagudo, C. A significant percentage of CD20-positive TILs correlates with poor prognosis in patients with primary cutaneous malignant melanoma. Histopathology 65, 726-728 (2014).

23. Miligy, I. et al. Prognostic significance of tumour infiltrating $B$ lymphocytes in breast ductal carcinoma in situ. Histopathology 71, 258-268 (2017).

24. Chevrier, S. et al. An immune atlas of clear cell renal cell carcinoma. Cell 169, 736-749 (2017). e718.

25. Corvigno, S. et al. Markers of fibroblast-rich tumor stroma and perivascular cells in serous ovarian cancer: Inter- and intra-patient heterogeneity and impact on survival. Oncotarget 7, 18573-18584 (2016).
26. Frodin, M. et al. Perivascular PDGFR-beta is an independent marker for prognosis in renal cell carcinoma. Br. J. Cancer 116, 195-201 (2017).

27. Gruen, B. \& Leisch, F. Fitting finite mixtures of generalized linear regressions in R. Comput. Stat. Data Anal. 51, 5247-5252 (2007).

28. Rolink, A. G., Schaniel, C., Busslinger, M., Nutt, S. L. \& Melchers, F. Fidelity and infidelity in commitment to B-lymphocyte lineage development. Immunol. Rev. 175, 104-111 (2000).

29. Xiao, X. et al. PD-1hi identifies a novel regulatory b-cell population in human hepatoma that promotes disease progression. Cancer Discov. 6, 546-559 (2016).

30. Lee, K. E. et al. Hif1a deletion reveals pro-neoplastic function of B cells in pancreatic neoplasia. Cancer Discov. 6, 256-269 (2016).

31. McDermott, D. F. et al. Atezolizumab, an anti-programmed death-ligand 1 antibody, in metastatic renal cell carcinoma: long-term safety, clinical activity, and immune correlates from a phase ia study. J. Clin. Oncol. 34, 833-842 (2016).

32. Young, R. M., Shaffer, A. L. 3rd, Phelan, J. D. \& Staudt, L. M. B-cell receptor signaling in diffuse large B-cell lymphoma. Semin. Hematol. 52, 77-85 (2015). 